Anna Esposito Nick Campbell Carl Vogel Amir Hussain Anton Nijholt (Eds.)

\title{
Development of Multimodal Interfaces: Active Listening and Synchrony
}

Second COST 2102 International Training School Dublin, Ireland, March 23-27, 2009 Revised Selected Papers 


\section{Preface}

This volume brings together, through a peer-revision process, the advanced research results obtained by the European COST Action 2102: Cross-Modal Analysis of Verbal and Nonverbal Communication, primarily discussed for the first time at the Second COST 2102 International Training School on "Development of Multimodal Interfaces: Active Listening and Synchrony" held in Dublin, Ireland, March 23-27 2009.

The school was sponsored by COST (European Cooperation in the Field of Scientific and Technical Research, www.cost.esf.org ) in the domain of Information and Communication Technologies (ICT) for disseminating the advances of the research activities developed within the COST Action 2102: "Cross-Modal Analysis of Verbal and Nonverbal Communication" (cost2102.cs.stir.ac.uk)

COST Action 2102 in its third year of life brought together about 60 European and 6 overseas scientific laboratories whose aim is to develop interactive dialogue systems and intelligent virtual avatars graphically embodied in a 2D and/or 3D interactive virtual world, capable of interacting intelligently with the environment, other avatars, and particularly with human users.

The main focus of the school was the development of multimodal interfaces. Traditional approaches to multimodal interface design tend to assume a "ping-pong" or "push-to-talk" approach to speech interaction wherein either the system or the human interlocutor is active at any one time. This is contrary to many recent findings in conversation and discourse analysis, where the definition of a "turn" or even an "utterance" is found to be very complex. People don't "take turns" to talk in a typical conversational interaction, but they each contribute actively to the joint emergence of a "common understanding." The sub-theme of the school was "Synchrony and Active Listening" selected with the idea to identify contributions that actively give support to the ongoing research into the dynamics of human spoken interaction, to the production of multimodal conversation data and to the subsequent analysis and modelling of interaction dynamics, with the dual goal of appropriately designing multimodal interfaces, as well as providing new approaches and developmental paradigms.

The themes of the papers presented in this book emphasize theoretical and practical issues for modelling human-machine interaction, ranging from the attempt in describing "the spacing and orientation in co-present interaction" to the effort for developing multimodal interfaces, collecting and analyzing interaction data and emergent behavior as well as analyzing the use of nonverbal and pragmatic elements of exchanges, implementing discourse control and virtual agents and using active listening in computer speech processing. The papers included in this book benefited from the live interactions in person among the many participants of the successful meeting in Dublin. Over 100 established and apprenticing researchers converged for the event.

The editors would like to thank the ESF COST- ICT Programme for its support in the realization of the school and the publication of this volume, and in particular the COST Science Officers Gian Mario Maggio, Francesca Boscolo, Bernie O’Neill, and Matteo Razzanelli for their constant help, guidance, and encouragement and 
Sietske Zeinstrafor for supporting and guiding the publication effort. That the event was successful owes this partly to more individuals than can be named, but notably: Marcus Furlong, Jean Maypother, Alena Moison. Our special appreciation goes to Gaetano Scarpetta, Dean of the International Institute for Advanced Scientific Studies, who supported and encouraged the editorial process including collecting, reviewing, and improving the manuscripts submitted. The IIASS team, Tina Marcella Nappi, Michele Donnarumma, and Antonio Natale, are acknowledged for their precious technical support in the organization of this volume.

In addition, the editors are grateful to the contributors for making this book a scientifically stimulating compilation of new and original ideas. Finally, the editors would like to express their greatest appreciation to all the members of the COST 2102 International Scientific Committee for their rigorous and invaluable scientific revisions, for their dedication, and their priceless selection process.

Anna Esposito

Nick Campbell

Carl Vogel

Amir Hussain

Anton Nijholt 